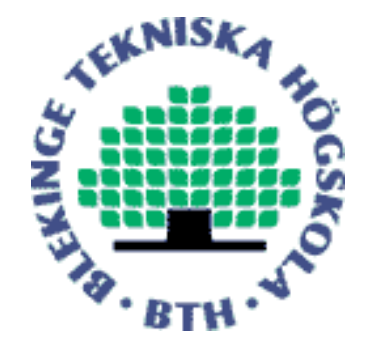

Copyright (C) 2014 IEEE.

Citation for the published paper:

Cognitive AF Relay Assisting both Primary and Secondary Transmission with Beamforming

Thi My Chinh Chu, Hoc Phan, Hans-Jürgen Zepernick

IEEE International Conference on Communications and Electronics (ICCE)

2014 Danang, Vietnam

This material is posted here with permission of the IEEE. Such permission of the IEEE does not in any way imply IEEE endorsement of any of BTH's products or services Internal or personal use of this material is permitted. However, permission to reprint/republish this material for advertising or promotional purposes or for creating new collective works for resale or redistribution must be obtained from the IEEE by sending a blank email message to pubs-permissions@iee.org.

By choosing to view this document, you agree to all provisions of the copyright laws protecting it. 


\title{
Cognitive AF Relay Assisting both Primary and Secondary Transmission with Beamforming
}

\author{
Thi My Chinh Chu, Hoc Phan, and Hans-Jürgen Zepernick \\ Blekinge Institute of Technology, SE-371 79 Karlskrona, Sweden \\ E-mail: $\{$ cch, hph, hjz $\} @$ bth.se
}

\begin{abstract}
This paper investigates the system performance of a cognitive relay network with underlay spectrum sharing wherein the relay is exploited to assist both the primary and secondary transmitters in forwarding their signals to the respective destinations. To exploit spatial diversity, beamforming transmission is implemented at the transceivers of the primary and secondary networks. Particularly, exact expressions for the outage probability and symbol error rate (SER) of the primary transmission and tight bounded expressions for the outage probability and SER of the secondary transmission are derived. Furthermore, an asymptotic analysis for the primary network, which is utilized to investigate the diversity and coding gain of the network, is developed. Finally, numerical results are presented to show the benefits of the proposed system.
\end{abstract}

\section{INTRODUCTION}

In recent years, incorporating cooperative transmission into cognitive radio networks (CRNs) has attracted great research interest due to its advantages such as high efficiency in spectrum utilization, reliable transmission, and large radio coverage (see [1] and the references therein). Considering the use of relay transmission in CRNs, the work of [2] investigated the outage performance of a cognitive amplify-and-forward (AF) relay network. Furthermore, the authors of [3] studied the integration of a decode-and-forward (DF) relaying scheme into a CRN by examining the outage performance. Moreover, the performance in terms of channel capacity of a cognitive cooperative radio network (CCRN) was analyzed in [4].

Regarding techniques of accessing the licensed spectrum for CRNs, there exist spectrum overlay, spectrum underlay, and interweave (opportunistic) schemes. For instance, in [5], several spectrum sensing techniques and requirements of designing an overlay CRN have been investigated. On the contrary, [6] investigated the outage probability of an underlay CRN under the interference power constraint of the primary user assuming perfect channel state information (CSI) for the interference channel from the cognitive transmitter to the primary receiver. Furthermore, the work of [7] investigated the benefits of using cooperative relays in interweave cognitive radio systems which provide cooperative diversity gain.

Recently, beamforming transmission has been proposed as an efficient technology to improve system performance and has been studied in the area of CRNs. In particular, the work of [8] investigated the implementation of beamforming transmission for underlay cognitive two-way relaying networks. In addition, the authors of [9] considered power allocation strategies for CRNs using beamforming transmission to guarantee the qual- ity of service (QoS) of the primary system when the secondary users (SUs) are given access to the licensed spectrum of the primary users (PUs). However, in [8], [9], the relays forward the signal of CRNs only. Theoretically, a terminal may receive signals from any neighbor transmitter due to the broadcast nature of wireless communications. Therefore, some recent transmission schemes in which the secondary relays also act as relays for the primary transmission have been proposed. For instance, considering the case of a singleantenna at all terminals of a cognitive AF relay system, it has been shown that letting the secondary relay forward the signals of both the primary and secondary transmitters results in a significant performance improvement for the primary network [10]. The main advantage of these schemes is that the interference incurred by the primary transmitter at the relay can be exploited to increase capacity for the primary network. As a result, the reliability of the primary transmission is enhanced. Further, these infrastructures become more efficient in utilizing spectrum and achieve better performance.

This paper utilizes beamforming transmission in a cognitive relay network wherein the secondary AF relay simultaneously forwards the primary and secondary signals to the respective destinations. All wireless channels are modeled as Nakagami$m$ fading which comprises a variety of fading models as special cases. We further investigate system performance by deriving exact and asymptotic expressions for the outage probability of the primary and secondary transmissions. Numerical results are presented showing that the primary transmission of the considered network outperforms that of the respective network without the assistance of the relay.

Notation: Vectors are denoted by bold lower case letters and their Frobenius norm is expressed as $\|\cdot\|_{F}$. Further, the probability density function (PDF) and cumulative distribution function $(\mathrm{CDF})$ of a random variable $(\mathrm{RV}) X$ are $f_{X}(\cdot)$ and $F_{X}(\cdot)$, respectively. Next, $C_{k}^{n}$ represents the binomial coefficient. Additionally, the gamma and incomplete gamma functions, defined in [11, eq. (8.310.1)] and [11, eq. (8.350.2)], are denoted as $\Gamma(n)$ and $\Gamma(n, x)$, respectively. Finally, $U(a, b ; x)$ stands for the confluent hypergeometric function [11, eq. (9.211.4)] and $\mathbb{E}\{\cdot\}$ is the expectation operator.

\section{SyStem AND Channel MODELS}

Consider an underlay cognitive relay network which coexists with a primary network over Nakagami- $m$ fading as depicted in Fig. 1. The secondary network consists of a 


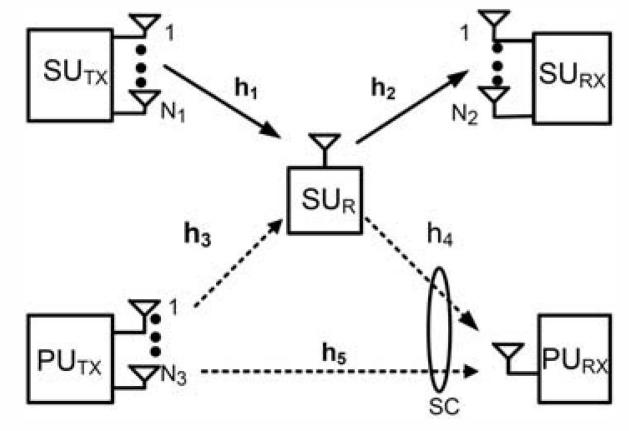

Fig. 1. System model of the consider system ( $\mathrm{SC}=$ Selection combining).

secondary transmitter $\mathrm{SU}_{\mathrm{TX}}$ with $N_{1}$ antennas, a secondary receiver $\mathrm{SU}_{\mathrm{RX}}$ with $N_{2}$ antennas, and a secondary relay $\mathrm{SU}_{\mathrm{R}}$ with a single receiver and transmit antenna. In view of the interference power constraint imposed by the primary user, $\mathrm{SU}_{\mathrm{TX}}$ needs to transmit with relatively low power. Furthermore, we assume that $\mathrm{SU}_{\mathrm{TX}}$ is located far from $\mathrm{SU}_{\mathrm{RX}}$. Therefore, the signal of the direct transmission from $\mathrm{SU}_{\mathrm{TX}}$ to $\mathrm{SU}_{\mathrm{RX}}$ can be neglected. In order to achieve spatial diversity for the cognitive network, we deploy beamforming at $\mathrm{SU}_{\mathrm{TX}}$ and maximum ratio combining at $\mathrm{SU}_{\mathrm{RX}}$. The primary network consists of a primary transmitter $\mathrm{PU}_{\mathrm{TX}}$ equipped with $N_{3}$ antennas and a primary receiver $\mathrm{PU}_{\mathrm{RX}}$ with a single antenna. The communication between $\mathrm{PU}_{\mathrm{TX}}$ and $\mathrm{PU}_{\mathrm{RX}}$ is mainly performed through the respective direct link and partially through the assistance of the cognitive relay. To focus the transmit signal towards the single receive antenna of $\mathrm{PU}_{\mathrm{RX}}$, we also employ beamforming transmission at $\mathrm{PU}_{\mathrm{TX}}$.

Because there is no dedicated feedback channel from $\mathrm{SU}_{\mathrm{TX}}$ to $\mathrm{PU}_{\mathrm{RX}}$, the instantaneous channel power gain of the link from $\mathrm{SU}_{\mathrm{TX}}$ to $\mathrm{PU}_{\mathrm{RX}}$ is unavailable at the $\mathrm{SU}_{\mathrm{TX}}$. However, $\mathrm{SU}_{\mathrm{TX}}$ can estimate the average channel gain for this channel based on some relatively stable parameters such as transmission distance and transmit/receive antenna gains. Utilizing this information, the $\mathrm{SU}_{\mathrm{TX}}$ controls its average transmit power to always meet the interference constraint at the $\mathrm{PU}_{\mathrm{RX}}$. With this assumption, the interference from the $\mathrm{SU}_{\mathrm{TX}}$ to the $\mathrm{PU}_{\mathrm{RX}}$ can be neglected. Thanks to the assistance of $\mathrm{SU}_{\mathrm{R}}$ in forwarding the primary signal, there exits a dedicated feedback channel from $\mathrm{PU}_{\mathrm{RX}}$ to $\mathrm{SU}_{\mathrm{R}}$, i.e., the instantaneous channel power gain of the link from $\mathrm{SU}_{\mathrm{R}}$ to $\mathrm{PU}_{\mathrm{RX}}$ is available at $\mathrm{SU}_{\mathrm{R}}$. Let $x_{s}$ and $x_{p}$ be, respectively, the transmit signals at $\mathrm{SU}_{\mathrm{TX}}$ and $\mathrm{PU}_{\mathrm{TX}}$ with corresponding average transmit powers $P_{p}=E\left\{\left|x_{p}\right|^{2}\right\}$ and $P_{s}=E\left\{\left|x_{s}\right|^{2}\right\}$. Before transmitting on the $i$-th antenna, the transmit signal at $\mathrm{SU}_{\mathrm{TX}}$ is weighted by a complex number $w_{1, i}=h_{1, i} /\left\|\mathbf{h}_{1}\right\|_{F}$ where $h_{1, i}$ is the channel coefficient of the link from the $i$-th transmit antenna at $\mathrm{SU}_{\mathrm{TX}}$ to the receive antenna at $\mathrm{SU}_{\mathrm{R}}$. Here, $\mathbf{h}_{1}$ is a $1 \times N_{1}$ fading channel coefficient vector of the channel from $\mathrm{SU}_{\mathrm{TX}}$ to $\mathrm{SU}_{\mathrm{R}}$ whose elements are modeled as Nakagami- $m$ fading with fading severity parameter $m_{1}$ and channel mean power $\Omega_{1}$. Then, a $1 \times N_{1}$ beamforming vector $\mathbf{w}_{1}$ at $\mathrm{SU}_{\mathrm{TX}}$ is constructed by collecting the weights for all antennas, $\mathbf{w}_{1}=\mathbf{h}_{1} /\left\|\mathbf{h}_{1}\right\|_{F}$. Similarly, a $1 \times N_{3}$ beamforming vector at $\mathrm{PU}_{\mathrm{TX}}$ for the primary transmission is selected as $\mathbf{w}_{5}=\mathbf{h}_{5} /\left\|\mathbf{h}_{5}\right\|_{F}$, where $\mathbf{h}_{5}$ is a $1 \times N_{3}$ fading channel coefficient vector of the channel from $\mathrm{PU}_{\mathrm{TX}}$ to $\mathrm{PU}_{\mathrm{RX}}$ whose elements are modeled as Nakagami- $m$ fading with fading severity parameter $m_{5}$ and channel mean power $\Omega_{5}$. As a consequence, in the first hop, the received signal $y_{r}$ at $\mathrm{SU}_{\mathrm{R}}$ and $y_{p_{1}}$ at $\mathrm{PU}_{\mathrm{RX}}$ are, respectively, given as

$$
\begin{aligned}
& y_{r}=\mathbf{h}_{1} \mathbf{w}_{1}^{H} x_{s}+\mathbf{h}_{3} \mathbf{w}_{5}^{H} x_{p}+n_{r} \\
& y_{p_{1}}=\mathbf{h}_{5} \mathbf{w}_{5}^{H} x_{p}+n_{p_{1}}
\end{aligned}
$$

where $\mathbf{h}_{3}$ is a $1 \times N_{3}$ fading channel coefficient vector of the channel from $\mathrm{PU}_{\mathrm{TX}}$ to $\mathrm{SU}_{\mathrm{R}}$ whose elements are modeled as Nakagami- $m$ fading with fading severity $m_{3}$ and channel mean power $\Omega_{3}$. Moreover, $n_{r}$ and $n_{p_{1}}$ are, respectively, the additive white Gaussian noises (AWGNs) at $\mathrm{SU}_{\mathrm{R}}$ and $\mathrm{PU}_{\mathrm{RX}}$ with zero mean and variance $N_{0}$. At $\mathrm{SU}_{\mathrm{R}}$, the received signal is then amplified with a factor $G$ and forwarded to $\mathrm{SU}_{\mathrm{RX}}$. On one hand, $\mathrm{SU}_{\mathrm{R}}$ must control its transmit power to keep the interference power from the secondary transmission at $\mathrm{PU}_{\mathrm{RX}}$ less than a predefined threshold $Q$. On the other hand, $\mathrm{PU}_{\mathrm{TX}}$ expects to increase the transmit power to achieve sufficient signal-to-noise ratio (SNR). To compromise these conditions, the amplifying gain $G$ is selected to maintain equilibrium as $E\left\{\left|G \mathbf{h}_{1} \mathbf{w}_{1}^{H} x_{s}\right|^{2}\right\}=Q /\left|h_{4}\right|^{2}$, or $G^{2}=Q / P_{s}\left\|\mathbf{h}_{1}\right\|_{F}^{2}\left|h_{4}\right|^{2}$. Here, $h_{4}$ is the channel coefficient from $\mathrm{SU}_{\mathrm{R}}$ to $\mathrm{PU}_{\mathrm{RX}}$ with fading severity parameter $m_{4}$ and channel mean power $\Omega_{4}$. Therefore, the received signals at $\mathrm{SU}_{\mathrm{RX}}$ and $\mathrm{PU}_{\mathrm{RX}}$ are, respectively, given by

$$
\begin{aligned}
& y_{s}=G \mathbf{w}_{2}^{H} \mathbf{h}_{2} \mathbf{h}_{1} \mathbf{w}_{1}^{H} x_{s}+G \mathbf{w}_{2}^{H} \mathbf{h}_{2} \mathbf{h}_{3} \mathbf{w}_{5}^{H} x_{p}+G \mathbf{w}_{2}^{H} \mathbf{h}_{2} n_{r}+n_{s} \\
& y_{p_{2}}=G h_{4} \mathbf{h}_{1} \mathbf{w}_{1}^{H} x_{s}+G h_{4} \mathbf{h}_{3} \mathbf{w}_{5}^{H} x_{p}+G h_{4} n_{r}+n_{p_{2}}
\end{aligned}
$$

where $n_{s}$ and $n_{p_{2}}$ are, respectively, the AWGN at $\mathrm{SU}_{\mathrm{RX}}$ and $\mathrm{PU}_{\mathrm{RX}}$ with zero mean and variance $N_{0}$. Next, $\mathbf{h}_{2}$ is a $1 \times N_{2}$ fading channel coefficient vector of the channel from $\mathrm{SU}_{R}$ to $S_{R X}$ whose elements are Nakagami- $m$ fading with fading severity parameter $m_{2}$ and channel mean power $\Omega_{2}$. Finally, $\mathbf{w}_{2}$ is a $1 \times N_{2}$ beamforming vector at $\mathrm{SU}_{\mathrm{RX}}, \mathbf{w}_{2}=\mathbf{h}_{2} /\left\|\mathbf{h}_{2}\right\|_{F}$. As observed from the system model in Fig. 1, the primary and secondary transmissions affect each other. In (3), the terms $G \mathbf{w}_{2}^{H} \mathbf{h}_{2} \mathbf{h}_{3} \mathbf{w}_{5}^{H} x_{p}$ and $G \mathbf{w}_{2}^{H} \mathbf{h}_{2} \mathbf{h}_{1} \mathbf{w}_{1}^{H} x_{s}$ are the interference of the secondary and primary networks, respectively. Therefore, the instantaneous signal-to-interference plus noise ratio (SINR) at $\mathrm{SU}_{\mathrm{RX}}, \gamma_{S}$, can be obtained from (3) as

$$
\gamma_{S}=\frac{X_{1} X_{2}}{a X_{2} X_{3}+b X_{1} X_{4}+c X_{2}}
$$

where $X_{l}=\left\|\mathbf{h}_{l}\right\|_{F}^{2}, l=1,2,3$ and $X_{4}=\left|h_{4}\right|^{2}, a=\frac{P_{p}}{P_{s}}, b=$ $\frac{N_{0}}{Q}$, and $c=\frac{N_{0}}{P_{s}}$. The instantaneous SNR of the direct link $\mathrm{PU}_{\mathrm{TX}} \rightarrow \mathrm{PU}_{\mathrm{RX}}, \gamma_{P_{1}}$, and the instantaneous SINR of the relaying link $\mathrm{PU}_{\mathrm{TX}} \rightarrow \mathrm{SU}_{R} \rightarrow \mathrm{PU}_{\mathrm{RX}}, \gamma_{P_{2}}$, are, respectively, determined from (2) and (4) as

$$
\begin{aligned}
& \gamma_{P_{1}}=g X_{5} \\
& \gamma_{P_{2}}=X_{3} /\left(d X_{1}+f\right)
\end{aligned}
$$


where $X_{5}=\left\|\mathbf{h}_{5}\right\|_{F}^{2}, d=\frac{P_{s}}{P_{p}}\left(1+\frac{N_{0}}{Q}\right), f=\frac{N_{0}}{P_{P} Q}$, and $g=P_{p} / N_{0}$. Given $G^{2}=\frac{Q}{P_{s}\left\|\mathbf{h}_{1}\right\|_{F}^{2}\left|h_{4}\right|^{2}}$, then $X_{4}$ does not appear in (7). It is noted that $X_{l}, l \in\{1,2,3,5\}$, is formulated as the summation of independent and identically distributed gamma RVs. Thus, $X_{1}, X_{2}, X_{3}$, and $X_{5}$ are, respectively, gamma distributed RVs with parameter sets $\left(N_{1} m_{1}, \alpha_{1}^{-1}\right)$, $\left(N_{2} m_{2}, \alpha_{2}^{-1}\right),\left(N_{3} m_{3}, \alpha_{3}^{-1}\right)$, and $\left(N_{3} m_{5}, \alpha_{5}^{-1}\right)$ with $\alpha_{l}=$ $\frac{m_{l}}{\Omega_{l}}$. Note that a gamma distributed RV $X$ with parameters $\left(m, \alpha^{-1}\right)$, where $m$ is a positive integer, has, respectively, the $\mathrm{PDF}$ and $\mathrm{CDF}$ as

$$
\begin{gathered}
f_{X}(x)=\frac{\alpha^{m}}{\Gamma(m)} x^{m-1} \exp (-\alpha x) \\
F_{X}(x)=1-\exp (-\alpha x) \sum_{p=0}^{m-1} \frac{\alpha^{p} x^{p}}{p !}
\end{gathered}
$$

\section{Outage Probability}

\section{A. Outage Probability of the Primary Network}

Assume that selection combining (SC) is applied at $\mathrm{PU}_{\mathrm{RX}}$ to select the signal between the relaying and direct transmission of the primary system. Therefore, the instantaneous end-toend SNR of the primary transmission can be attained as $\gamma_{P}=$ $\max \left(\gamma_{P_{1}}, \gamma_{P_{2}}\right)$. Utilizing the order statistics theory, the CDF of $\gamma_{P}$ is given by

$$
F_{\gamma_{P}}(\gamma)=F_{\gamma_{P_{1}}}(\gamma) F_{\gamma_{P_{2}}}(\gamma)
$$

From (6) and (7), $F_{\gamma_{\boldsymbol{P}_{1}}}(\gamma)$ and $F_{\gamma_{\boldsymbol{P}_{2}}}(\gamma)$ are, respectively, determined as

$$
\begin{aligned}
& F_{\gamma_{P_{1}}}(\gamma)=1-\exp \left(-\frac{\alpha_{5} \gamma}{g}\right) \sum_{k=0}^{N_{3} m_{5}-1} \frac{\alpha_{5}^{k} \gamma^{k}}{k ! g^{k}} \\
& F_{\gamma_{P_{2}}}(\gamma)=\int_{0}^{\infty} F_{X_{3}}\left[\gamma\left(d \cdot x_{1}+f\right)\right] f_{X_{1}}\left(x_{1}\right) d x_{1}
\end{aligned}
$$

From (9) together with the binomial theorem [11, eq. (1.111)], we obtain an expression for $F_{X_{3}}\left[\gamma\left(d \cdot x_{1}+e\right)\right]$. Substituting this outcome and the expression of $f_{X_{1}}\left(x_{1}\right)$ given in (8) into (12), we have

$$
\begin{aligned}
& F_{\gamma_{\boldsymbol{P}_{2}}}(\gamma)=1-\exp \left(-f \alpha_{3} \gamma\right) \sum_{l=0}^{N_{3} m_{3}-1} \frac{\alpha_{3}^{l} \gamma^{l}}{l !} \sum_{i=0}^{l} \frac{C_{i}^{l} d^{i} f^{l-i}}{\Gamma\left(N_{1} m_{1}\right)} \\
& \times \alpha_{1}^{N_{1} m_{1}} \int_{0}^{\infty} x_{1}^{N_{1} m_{1}+i-1} \exp \left(-\left(\alpha_{1}+\alpha_{3} d \gamma\right) x_{1}\right) d x_{1}
\end{aligned}
$$

Then, applying [11, eq. (3.381.4)] to solve the remaining integral of (13), we obtain $F_{\gamma_{\boldsymbol{P}_{2}}}(\gamma)$ as

$$
\begin{aligned}
& F_{\gamma_{\boldsymbol{P}_{2}}}(\gamma)=1-\sum_{l=0}^{N_{3} m_{3}-1} \sum_{i=0}^{l} \frac{C_{i}^{l} \Gamma\left(N_{1} m_{1}+i\right) \alpha_{1}^{N_{1} m_{1}} f^{l-i}}{l ! \Gamma\left(N_{1} m_{1}\right) \alpha_{3}^{N_{1} m_{1}+i-l} d^{N_{1} m_{1}}} \\
& \times\left(\gamma+\frac{\alpha_{1}}{\alpha_{3} d}\right)^{-N_{1} m_{1}-i} \gamma^{l} \exp \left(-f \alpha_{3} \gamma\right)
\end{aligned}
$$

Substituting (14) and (11) into (10), an exact expression for the $\mathrm{CDF}$ of $\gamma_{P}$ is given by

$$
\begin{aligned}
& F_{\gamma_{\boldsymbol{P}}}(\gamma)=1-\sum_{l=0}^{N_{3} m_{3}-1} \sum_{i=0}^{l} \frac{C_{i}^{l} \Gamma\left(N_{1} m_{1}+i\right) \alpha_{1}^{N_{1} m_{1}} f^{l-i}}{l ! \Gamma\left(N_{1} m_{1}\right) \alpha_{3}^{N_{1} m_{1}+i-l} d^{N_{1} m_{1}}} \\
& \times \frac{\gamma^{l} \exp \left(-f \alpha_{3} \gamma\right)}{\left(\gamma+\frac{\alpha_{1}}{\alpha_{3} d}\right)^{N_{1} m_{1}+i}}-\sum_{k=0}^{N_{3} m_{5}-1} \frac{\alpha_{5}^{k} \gamma^{k}}{k ! g^{k}} \exp \left(-\alpha_{5} \frac{\gamma}{g}\right) \\
& +\sum_{l=0}^{N_{3} m_{3}-1} \sum_{i=0}^{l} \frac{C_{i}^{l} \Gamma\left(N_{1} m_{1}+i\right)}{l ! \Gamma\left(N_{1} m_{1}\right)} \sum_{k=0}^{N_{3} m_{5}-1} \frac{1}{k !} \frac{\alpha_{1}^{N_{1} m_{1}} \alpha_{5}^{k} f^{l-i}}{\alpha_{3}^{N_{1} m_{1}+i-l}} \\
& \times \frac{\left(\gamma+\frac{\alpha_{1}}{\alpha_{3} d}\right)^{-N_{1} m_{1}-i}}{d^{N_{1} m_{1}} g^{k}} \gamma^{l+k} \exp \left(-\frac{f g \alpha_{3}+\alpha_{5}}{g} \gamma\right)
\end{aligned}
$$

Outage probability is defined as the probability that the instantaneous SNR falls below a predefined threshold $\gamma_{t h}^{P}$. The outage probability of the primary transmission can be calculated with (15) as $P_{\text {out }}=F_{\gamma_{\boldsymbol{p}}}\left(\gamma_{t h}^{P}\right)$.

\section{B. Outage Probability of the Secondary Network}

It can clearly be seen from (5) that the instantaneous SINR of the secondary transmission is mathematically represented by a complicated function of multiple independent RVs, i.e., $X_{i}, i=1,2,3,4$. Thus, finding an exact expression for $F_{\gamma_{S}}(\gamma)$, from (5) can be very difficult. Taking advantage of [12, eq. (25)], we adopt a tightly upper bounded expression $\gamma_{S U}$ for $\gamma_{S}$ as

$$
\gamma_{S U}=\min \left(\gamma_{S_{1}}, \gamma_{S_{2}}\right)
$$

where $\gamma_{S_{1}}=X_{1} /\left(a X_{3}+c\right)$ and $\gamma_{S_{2}}=X_{2} /\left(b X_{4}\right)$. Since $X_{1}, X_{2}, X_{3}$, and $X_{4}$ are independent, $\gamma_{S_{1}}$ and $\gamma_{S_{2}}$ are also mutually independent. As a result, the lower bounded CDF of $\gamma_{S}$ can be derived from the CDFs of $\gamma_{S_{1}}$ and $\gamma_{S_{2}}$ based on the order statistics theory as

$$
F_{\gamma_{S U}}(\gamma)=1-\left[1-F_{\gamma_{S_{1}}}(\gamma)\right]\left[1-F_{{\gamma_{S_{2}}}}(\gamma)\right]
$$

where

$$
\begin{aligned}
& F_{\gamma_{S_{1}}}(\gamma)=\int_{0}^{\infty} F_{X_{1}}\left[\gamma\left(a x_{3}+c\right)\right] f_{X_{3}}\left(x_{3}\right) d x_{3} \\
& F_{\gamma_{S_{2}}}(\gamma)=\int_{0}^{\infty} F_{X_{2}}\left(b \gamma x_{4}\right) f_{X_{4}}\left(x_{4}\right) d x_{4}
\end{aligned}
$$

Using (9) along with the binomial theorem [11, eq. (1.111)], we get $F_{X_{1}}\left(\gamma\left(a x_{3}+c\right)\right)$. Next, substituting this outcome and $f_{X_{3}}\left(x_{3}\right)$ given in (8) into (18); the expression of $F_{X_{2}}\left(b \gamma x_{4}\right)$ given in (9) and $f_{X_{4}}\left(x_{4}\right)$ given in (8) into (19), then applying [11, eq. (3.381.4)] to solve the remaining integrals, expressions for the CDF of $\gamma_{S_{1}}$ and $\gamma_{S_{2}}$ are obtained as

$$
\begin{aligned}
& F_{\gamma_{S_{1}}}(\gamma)=1-\exp \left(-\alpha_{1} c \gamma\right) \sum_{p=0}^{N_{1} m_{1}-1} \sum_{i=0}^{p} \frac{C_{i}^{p}}{p !} \frac{c^{p-i} \alpha_{3}^{N_{3} m_{3}}}{a^{N_{3} m_{3}} \alpha_{1}^{N_{3} m_{3}-p+i}} \\
& \times \frac{\Gamma\left(N_{3} m_{3}+i\right)}{\Gamma\left(N_{3} m_{3}\right)} \frac{\gamma^{p}}{\left(\gamma+\alpha_{3} / a \alpha_{1}\right)^{N_{3} m_{3}+i}}
\end{aligned}
$$


$F_{\gamma_{S_{2}}}(\gamma)=1-\sum_{q=0}^{N_{2} m_{2}-1} \frac{1}{q !} \frac{\Gamma\left(m_{4}+q\right)}{\Gamma\left(m_{4}\right)} \frac{\alpha_{4}^{m_{4}}}{\alpha_{2}^{m_{4}} b^{m_{4}}} \frac{\gamma^{q}}{\left(\gamma+\frac{\alpha_{4}}{\alpha_{2} b}\right)^{m_{4}+q}}$

Finally, substituting (20) and (21) into (17), an expression for the CDF of $\gamma_{S U}$ can be written as

$$
\begin{aligned}
& F_{\gamma_{S U}}(\gamma)=1-\sum_{p=0}^{N_{1} m_{1}-1} \sum_{i=0}^{p} \sum_{q=0}^{N_{2} m_{2}-1} \frac{C_{i}^{p}}{p ! q !} \frac{\Gamma\left(m_{4}+q\right)}{\Gamma\left(m_{4}\right)} \\
& \times \frac{\Gamma\left(N_{3} m_{3}+i\right)}{\Gamma\left(N_{3} m_{3}\right)} \frac{c^{p-i}}{a^{N_{3} m_{3}} b^{m_{4}}} \frac{\alpha_{3}^{N_{3} m_{3}} \alpha_{4}^{m_{4}}}{\alpha_{1}^{N_{3} m_{3}-p+i}} \alpha_{2}^{m_{4}} \\
& \times \frac{\gamma^{p+q}}{\left(\gamma+\frac{\alpha_{3}}{a \alpha_{1}}\right)^{N_{3} m_{3}+i}\left(\gamma+\frac{\alpha_{4}}{\alpha_{2} b}\right)^{m_{4}+q}} \exp \left(-c \alpha_{1} \gamma\right)
\end{aligned}
$$

Therefore, outage probability of the secondary transmission can be directly obtained by using the outage threshold, $\gamma_{t h}^{S}$, as the argument for the expression in (22), $P_{o u t}^{S}=F_{\gamma_{S}}\left(\gamma_{t h}^{S}\right)$.

\section{ASYMPtotic AnAlysis FOR the PRIMARy NETWORK}

As can be seen from (15), the exact expression for the outage probability of the primary network is too complicated to render insights into the system performance. Thus, asymptotic expressions for the outage probability and SER in the high SNR regime are derived. In principle, the MacLaurin expansion for the CDF of the end-to-end instantaneous SNR, $\gamma_{P}$, at zero is deployed to analyze the asymptotic performance. Thus, we now adopt the MacLaurin expansion for the CDFs of $\gamma_{P_{1}}$ and $\gamma_{P_{2}}$, then substituting these outcomes into (10) to achieve an asymptotic expression for $\gamma_{P}$. From (6), we have $F_{\gamma_{\boldsymbol{P}_{1}}}(\gamma)=F_{X_{5}}(\gamma / g)$. Utilizing the MacLaurin expansion, in the high SNR regime, $f_{X_{5}}\left(x_{5}\right)$ is approximated as

$$
f_{X_{5}}\left(x_{5}\right)=\frac{\alpha_{5}^{N_{3} m_{5}}}{\Gamma\left(N_{3} m_{5}\right)} x_{5}^{N_{3} m_{5}-1}+o\left(x_{5}^{N_{3} m_{5}-1}\right)
$$

Hence, we obtain an approximation for $F_{X_{5}}\left(x_{5}\right)$ as

$$
F_{X_{5}}\left(x_{5}\right)=\frac{\alpha_{5}^{N_{3} m_{5}}}{\Gamma\left(N_{3} m_{5}+1\right)} x_{5}^{N_{3} m_{5}}+o\left(x_{5}^{N_{3} m_{5}}\right)
$$

Consequently, an asymptotic expression for $F_{\gamma_{\boldsymbol{P}_{1}}}(\gamma)$ is found as

$$
F_{\gamma_{\boldsymbol{P}_{1}}}^{\infty}(\gamma)=\frac{\alpha_{5}^{N_{3} m_{5}}}{\Gamma\left(N_{3} m_{5}+1\right)} \frac{\gamma^{N_{3} m_{5}}}{g^{N_{3} m_{5}}}
$$

In addition, taking the derivative of both sides of (12), the $n$-th order derivative of $F_{\gamma_{\boldsymbol{P}_{2}}}(\gamma)$ at zero is given by

$$
\left.F_{\gamma_{\boldsymbol{P}_{2}}}^{n}(\gamma)\right|_{\gamma=0}=\left.\int_{0}^{\infty} \frac{\partial^{n}\left[F_{X_{3}}\left(\Psi\left(x_{1}, \gamma\right)\right)\right]}{\partial \gamma^{n}}\right|_{\gamma=0} f_{X_{1}}\left(x_{1}\right) d x_{1}
$$

where $\Psi\left(x_{1}, \gamma\right)=\gamma\left(d \cdot x_{1}+e\right)$. Utilizing the similar approach, an asymptotic expression for $F_{X_{3}}\left(x_{3}\right)$ in the high SNR regime can be expressed as

$$
F_{X_{3}}\left(x_{3}\right)=\frac{\alpha_{3}^{N_{3} m_{3}}}{\Gamma\left(N_{3} m_{3}+1\right)} x^{N_{3} m_{3}}
$$

Then, applying [11, eq. $(0.430 .1)]$, the $n$-th order derivative of $F_{X_{3}}\left(\Psi\left(x_{1}, \gamma\right)\right)$ with respect to $\gamma$ at zero can be given by

$$
\begin{aligned}
& \left.\frac{\partial^{n}\left[F_{X_{3}}\left(\Psi\left(x_{1}, \gamma\right)\right)\right]}{\partial \gamma^{n}}\right|_{\gamma=0}=\sum_{u=1}^{n} \sum_{v=0}^{u-1} C_{v}^{u} \frac{(-1)^{v}\left(\Psi\left(x_{1}, \gamma\right)\right)^{v}}{\Gamma(u+1)} \\
& \times\left.\frac{\partial^{u} F_{X_{3}}\left(\Psi\left(x_{1}, \gamma\right)\right)}{\partial \Psi^{u}} \frac{\partial^{n}\left(\Psi\left(x_{1}, \gamma\right)\right)^{u-v}}{\partial \gamma^{n}}\right|_{\gamma=0}
\end{aligned}
$$

It can be easily seen that

$$
\begin{aligned}
& \left.\left(\Psi\left(x_{1}, \gamma\right)\right)^{v}\right|_{\gamma=0}= \begin{cases}\left.\left(\gamma\left(d x_{1}+e\right)\right)^{v}\right|_{\gamma=0}=0 ; & \text { if } v>0 \\
\left.\left(\gamma\left(d x_{1}+e\right)\right)^{\gamma}\right|_{\gamma=0}=1 ; & \text { if } v=0\end{cases} \\
& \left.\frac{\partial^{u} F_{X_{3}}\left(\Psi\left(x_{1}, \gamma\right)\right)}{\partial \Psi^{u}}\right|_{\gamma=0}= \begin{cases}\alpha_{3}^{N_{3} m_{3}} ; & \text { if } u=N_{3} m_{3} \\
0 ; & \text { if } u<N_{3} m_{3}\end{cases}
\end{aligned}
$$

Considering the case $v=0$ and $u=N_{3} m_{3}$, we have

$$
\begin{aligned}
& \left.\frac{\partial^{n}\left(\Psi\left(x_{1}, \gamma\right)\right)^{u-v}}{\partial \gamma^{n}}\right|_{\gamma=0}= \\
& \begin{cases}\Gamma\left(N_{3} m_{3}+1\right) \sum_{t=1}^{N_{3} m_{3}} C_{t}^{N_{3} m_{3}} d^{t} x_{1}^{t} e^{N_{3} m_{3}-t} ; & \text { if } n=N_{3} m_{3} \\
0 ; & \text { if } n<N_{3} m_{3}\end{cases}
\end{aligned}
$$

By substituting (29), (30), and (31) into (28), the $n$-th order derivative of $F_{X_{3}}\left(\Psi\left(x_{1}, \gamma\right)\right)$ at $\gamma=0$ is found as

$$
\begin{aligned}
& \left.\frac{\partial^{n}\left[F_{X_{3}}\left(\Psi\left(x_{1}, \gamma\right)\right)\right]}{\partial \gamma^{n}}\right|_{\gamma=0}= \\
& \begin{cases}\alpha_{3}^{N_{3} m_{3}} \sum_{t=1}^{N_{3} m_{3}} C_{t}^{N_{3} m_{3}} d^{t} x_{1}^{t} e^{N_{3} m_{3}-t} & \text { if } n=u=N_{3} m_{3} \\
0 & \text { if } u<N_{3} m_{3}\end{cases}
\end{aligned}
$$

Putting (8) and (32) into (26) along with the help of [11, eq. (3.381.4)] to solve the remaining integral, we obtain the $n$-th order derivative of $F_{\gamma_{\boldsymbol{P}_{2}}}(\gamma)$ at $\gamma=0$ as

$\left.F_{\gamma_{P_{2}}}^{(n)}(\gamma)\right|_{\gamma=0}=\left\{\begin{array}{lr}\sum_{t=1}^{N_{3} m_{3}} C_{t}^{N_{3} m_{3}} d^{t} e^{N_{3} m_{3}-t} \frac{\Gamma\left(N_{1} m_{1}+t\right)}{\Gamma\left(N_{1} m_{1}\right)} \frac{\alpha_{3}^{N_{3} m_{3}}}{\alpha_{1}^{N_{1} m_{1}}} \\ \text { if } n=N_{3} m_{3} \\ 0 & \text { if } n<N_{3} m_{3}\end{array}\right.$

Thus, an asymptotic expression for $F_{\gamma_{\boldsymbol{P}_{2}}}(\gamma)$ in the high SNR regime is given by

$$
\begin{aligned}
F_{\gamma_{2}}^{\infty}(\gamma) & =\sum_{t=0}^{N_{3} m_{3}} C_{t}^{N_{3} m_{3}} d^{t} e^{N_{3} m_{3}-t} \frac{\Gamma\left(N_{1} m_{1}+t\right)}{\Gamma\left(N_{1} m_{1}\right)} \\
& \times \frac{\alpha_{3}^{N_{3} m_{3}}}{\alpha_{1}^{N_{1} m_{1}}} \frac{\gamma^{N_{3} m_{3}}}{\Gamma\left(N_{3} m_{3}+1\right)}
\end{aligned}
$$

Substituting (25) and (35) into (10), an asymptotic expression for the instantaneous SINR of the primary transmission can 
be finally established as

$$
\begin{aligned}
& F_{\gamma_{\boldsymbol{P}}}^{\infty}(\gamma)=\sum_{t=0}^{N_{3} m_{3}} C_{t}^{N_{3} m_{3}} \frac{d^{t} f^{N_{3} m_{3}-t}}{g^{N_{3} m_{5}}} \frac{\Gamma\left(N_{1} m_{1}+t\right)}{\Gamma\left(N_{1} m_{1}\right)} \\
& \times \frac{\alpha_{3}^{N_{3} m_{3}} \alpha_{5}^{N_{3} m_{5}}}{\alpha_{1}^{N_{1} m_{1}}} \frac{\gamma^{N_{3} m_{5}+N_{3} m_{3}}}{\Gamma\left(N_{3} m_{3}+1\right) \Gamma\left(N_{3} m_{5}+1\right)}
\end{aligned}
$$

Now, we can obtain an asymptotic expression for the outage probability as $P_{\text {out }}^{P, \infty}=F_{\gamma_{\boldsymbol{P}}}\left(\gamma_{t h}^{P}\right)$. As pointed out in [13], in order to quantify the diversity gain of the system, we need to transform the expression of the outage probability of the primary system into the following form

$$
F_{\gamma_{P}}^{\infty}(\gamma)=\left(G_{c} \rho\right)^{-G_{d}}+o\left(\rho^{-G_{d}}\right)
$$

where $\rho=\frac{P_{p}}{N_{0}}$ denotes the average SNR of the primary system and $o\left(\rho^{-G_{\boldsymbol{d}}}\right)$ stands for the higher order terms of $F_{\gamma_{\boldsymbol{P}}}^{\infty}$, i.e., $\lim _{\rho \rightarrow \infty} \frac{o\left(\rho^{-G_{d}}\right)}{F_{\gamma P}^{\infty}}=0$. Furthermore, $G_{d}$ and $G_{c}$ are the diversity and coding gain of the primary system, respectively. In cooperative systems, $G_{d}$ is a crucial performance metric which determines the slope of the curve of the outage probability or SER versus average SNR on a log scale. Furthermore, the coding gain of the network, $G_{c}$, determines the shift of the asymptotic outage probability curve as compared to the benchmark curve $\rho^{-G_{d}}$. It is straightforward to see from (36) that the diversity gain of the primary system is $G_{d}=N_{3} m_{5}+N_{3} m_{3}$.

For the secondary network, the performance does not only depend on its transmit power at $\mathrm{SU}_{\mathrm{TX}}$ but also the interference power threshold $Q$ at $\mathrm{PU}_{\mathrm{RX}}$. With a fixed transmit power at $\mathrm{SU}_{\mathrm{TX}}$, for low value of $Q$, the outage probability of the secondary network will decrease corresponding to the increase of $Q$. However, when the value of $Q$ exceeds a threshold, for which the transmit power of $\mathrm{SU}_{\mathrm{TX}}$ and $\mathrm{SU}_{\mathrm{R}}$ always satisfy the interference constraint, the outage probability of the secondary network is almost invariant to the increase of $Q$ (see Fig. 4 and Fig. 5). Thus, there is no slope of the curve of the outage probability versus $Q / N_{0}$ in a $\log$ scale. Therefore, we do not investigate asymptotic performance for the secondary system.

\section{Numerical Results AND Discussions}

In this section, we present analytical results in comparison with Monte Carlo simulations. Let $d_{1}, d_{2}, d_{3}, d_{4}$, and $d_{5}$ be the normalized distances of the links $\mathrm{SU}_{\mathrm{TX}} \rightarrow \mathrm{SU}_{\mathrm{R}}$, $\mathrm{SU}_{\mathrm{R}} \rightarrow \mathrm{SU}_{\mathrm{RX}}, \mathrm{PU}_{\mathrm{TX}} \rightarrow \mathrm{SU}_{\mathrm{R}}, \mathrm{SU}_{\mathrm{R}} \rightarrow \mathrm{PU}_{\mathrm{RX}}$, and $\mathrm{PU}_{\mathrm{TX}} \rightarrow$ $\mathrm{PU}_{\mathrm{RX}}$, respectively. Assume that the path-loss decays with an exponent of 4 for highly shadowed urban environment.

First, we show the impact of fading severity parameters and the number of antennas at $\mathrm{PU}_{\mathrm{TX}}$ on the primary performance when fixing $d_{1}=d_{2}=d_{5}=0.5$ and $d_{2}=d_{3}=0.5$ in the following cases:

- Case 1: $N_{1}=N_{2}=2, N_{3}=2, m_{i}=0.5, i \in\{1, \cdots, 5\}$

- Case 2: $N_{1}=N_{2}=2, N_{3}=2, m_{i}=1.0, i \in\{1, \cdots, 5\}$

- Case 3: $N_{1}=N_{2}=2, N_{3}=3, m_{i}=1.0, i \in\{1, \cdots, 5\}$

The interference power-to-noise ratio $Q / N_{0}$ of $\mathrm{PU}_{\mathrm{RX}}$ and the average transmit power-to-noise ratio $P_{s} / N_{0}$ of $\mathrm{SU}_{\mathrm{TX}}$ are selected as $Q / N_{0}=5 \mathrm{~dB}$ and $P_{s} / N_{0}=10 \mathrm{~dB}$.

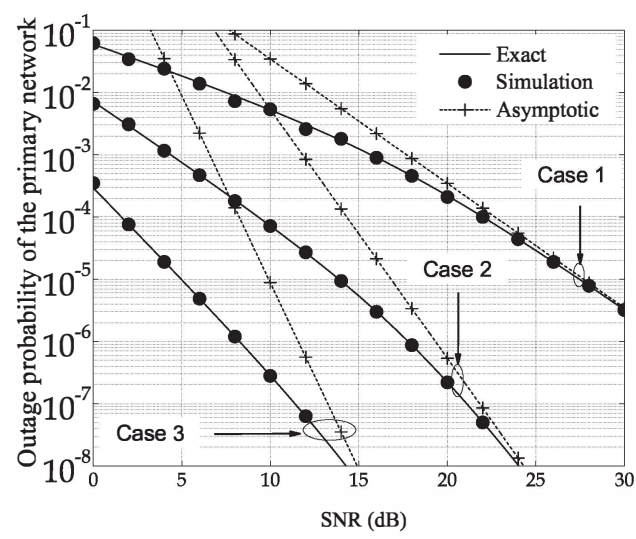

Fig. 2. Outage probability of the primary network for various fading severity parameters and different numbers of antennas at $\mathrm{PU}_{\mathrm{TX}}$.

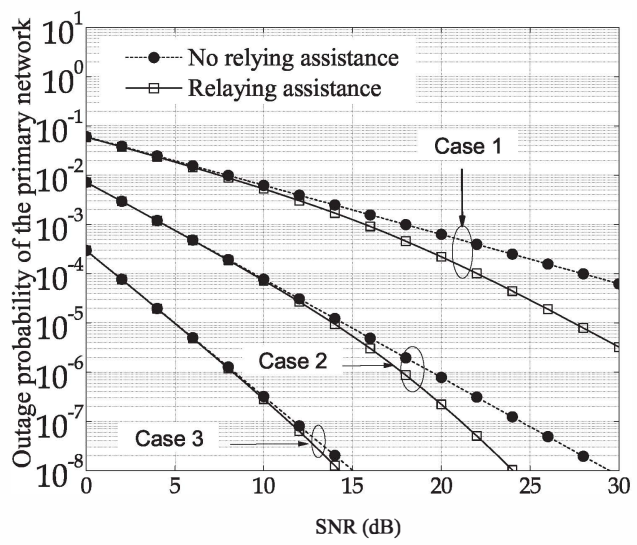

Fig. 3. Outage probability of the primary network with and without the assistance of $\mathrm{SU}_{\mathrm{R}}$.

For all the considered cases, the outage threshold is set as $\gamma_{t h}^{P}=\gamma_{t h}^{S}=3 \mathrm{~dB}$. As can be seen from Fig. 2 with the identical antenna configuration at the transceivers of Case 1 and Case 2, $N_{1}=N_{2}=N_{3}=2$, the primary performance in Case 2 is better than that in Case 1 . This is attributed to the fact

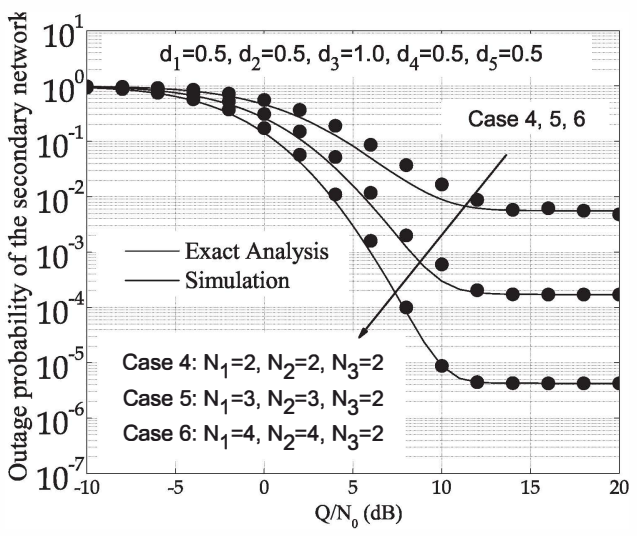

Fig. 4. Outage probability of the secondary network for different numbers of antennas at $\mathrm{SU}_{\mathrm{TX}}$ and $\mathrm{SU}_{\mathrm{RX}}$. 


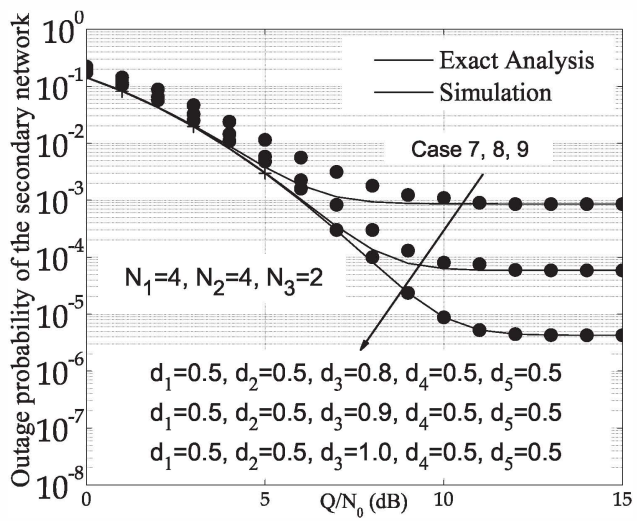

Fig. 5. Outage probability of the secondary network with various distances from $\mathrm{PU}_{\mathrm{TX}}$ to $\mathrm{SU}_{\mathrm{R}}$.

that Case 2 is endowed with more favorable fading conditions, $m_{l}=1, l \in\{1,2,3,4,5\}$, compared to Case $1, m_{l}=0,5$. Further, comparing Case 3 with Case 2, one can observe the effect of the number of antennas at $\mathrm{PU}_{\mathrm{TX}}$ on the outage probability and SER. Clearly, the outage probability decreases significantly relative to the increase in the number of antennas at the primary transmitter. As expected, the asymptotic curves converge to the analytical results as well as the simulations in the high SNR regime. From these plots, the achievable diversity gains for Case 1, Case 2, and Case 3 are observed as 2,4 , and 6 , respectively, which coincide with the asymptotic analysis.

Fig. 3 compares the outage probability of the primary networks with and without the assistance of $\mathrm{SU}_{\mathrm{R}}$. The network parameters are again selected as Case1, Case 2 and Case 3. As expected, in the high SNR regime, the performance of the primary network with the assistance of $\mathrm{SU}_{\mathrm{R}}$ outperforms that of the system without the assistance of $\mathrm{SU}_{R}$.

Fig. 4 exhibits the effect of the antenna configurations at $\mathrm{SU}_{\mathrm{TX}}$ and $\mathrm{SU}_{\mathrm{RX}}$ on the secondary performance. For these examples, the average transmit power-to-noise ratios $P_{p} / N_{0}$ at $\mathrm{PU}_{\mathrm{TX}}$ and $P_{s} / N_{0}$ at $\mathrm{SU}_{\mathrm{TX}}$ are selected as $P_{p} / N_{0}=P_{s} / N_{0}=$ $5 \mathrm{~dB}$. We change the number of antennas $N_{1}, N_{2}$ while fixing the other parameters, $d_{1}=d_{2}=d_{5}=0.5, d_{3}=1.0, N_{3}=2$, and $m_{l}=2, l \in\{1, \ldots, 5\}$. As observed from Fig. 4, when the number of antennas at the secondary transceivers increases, the outage probability of the secondary network decreases. Thus, employing multiple antennas together with beamforming transmission seems to be a suitable solution to improve the system performance of an underlay cognitive AF relay network which suffers from a strict constraint on its transmit power.

Fig. 5 depicts outage probability of the secondary transmission for various values of the distance from $\mathrm{PU}_{\mathrm{TX}}$ to $\mathrm{SU}_{\mathrm{R}}, d_{3}$, when fixing the number of antennas $N_{1}=N_{2}=4, N_{3}=2$ and distances $d_{1}=d_{2}=d_{4}=d_{5}=0.5$. The average transmit power-to-noise ratios $P_{p} / N_{0}$ and $P_{s} / N_{0}$ are selected as $P_{p} / N_{0}=P_{s} / N_{0}=5 \mathrm{~dB}$. It can be seen that when the distance between the primary transmitter and secondary relay become farther away, the secondary performance is improved.
This benefit can be attributed to the fact that the interference power imposed by the primary transmission to the secondary transmission reduces when this distance increases.

\section{CONCLUding REMARKS}

We have deployed beamforming transmission for the primary and secondary networks wherein the secondary relay facilitates both the primary and secondary communications. We have further analyzed the system performance of the network by deriving exact expressions for the outage probability of the primary network and tight bounded expressions for the outage probability of the secondary network. Moreover, an asymptotic analysis has been developed to reveal the diversity gain and coding gain of the primary network. Also, numerical examples have been presented to illustrate the impact of different network parameters on the system performance. The numerical results also indicate that the performance of the primary transmission of the considered network outperforms that of a network in which the secondary relay only assists the secondary transmission.

\section{REFERENCES}

[1] H. Luo, Z. Zhang, and G. Yu, "Cognitive cooperative relaying," in Proc. IEEE International Conference on Communications Systems, Singapore, Nov. 2008, pp. 1499-1503.

[2] T. M. C. Chu, H. Phan, and H.-J. Zepernick, "Performance analysis of MIMO cognitive amplify-and-forward relay networks with orthogonal space-time block codes," Wirel. Commun. Mob. Comput. DOI: 10.1002/wcm.2449, Jan. 2014.

[3] L. Luo, P. Zhang, G. Zhang, and J. Qin, "Outage performance for cognitive relay networks with underlay spectrum sharing," IEEE Commun. Lett., vol. 15, no. 7, pp. 710-712, Jul. 2011.

[4] S. Kim, W. Choi, Y. Choi, J. Lee, Y. Han, and I. Lee, "Downlink performance analysis of cognitive radio based cellular relay networks," in Proc. IEEE Cognitive Radio Oriented Wireless Networks and Communications, Singapore, May 2008, pp. 1-6.

[5] A. Ghasemi and E. Sousa, "Spectrum sensing in cognitive radio networks: requirements, challenges and design trade-offs," IEEE Commum. Magazine, vol. 46, no. 4, pp. 32-39, Apr. 2008.

[6] R. Zhang, "On peak versus average interference power constraints for protecting primary users in cognitive radio networks," IEEE Trans. Wireless. Commun., vol. 8, no. 4, pp. 2112-2120, Apr. 2009.

[7] A. A. Naguib, A. Elezabi, and M. Nafie, "A novel scheme for relay cooperation in interweave cognitive radio systems," in Proc. International Symposium on Applied Sciences in Biomedical and Communication Technologies, Rome, Italy, Nov. 2010, pp. 1-5.

[8] K. Jitvanichphaibool, Y.-C. Liang, and R. Zhang, "Beamforming and power control for multi-antenna cognitive two-way relaying," in Proc. IEEE Wireless Communications and Networking Conference, Budapest, Hungary, Apr. 2009, pp. 1-6.

[9] K. Zarifi, A. Ghrayeb, and S. Affes, "Jointly optimal source power control and relay matrix design in multipoint-to-multipoint cooperative communication networks," IEEE Trans. Signal Process., vol. 59, no. 9, pp. 4313-4330, Sep. 2011.

[10] T. M. C. Chu, H. Phan, and H.-J. Zepernick, "Amplify-and-forward relay assisting both primary and secondary transmissions in cognitive radio networks over Nakagami- $m$ fading," in Proc. IEEE International Symposium on Personal, Indoor and Mobile Radio Communications, Sydney, Australia, Sep. 2012.

[11] I. S. Gradshteyn and I. M. Ryzhik, Table of Integrals, Series, and Products, 7th ed. Academic Press, 2007.

[12] M. D. Renzo, F. Graziosi, and F. Santucci, "A comprehensive framework for performance analysis of dual-hop cooperative wireless systems with fixed-gain relays over generalized fading channels," IEEE Trans. Wireless Commun., vol. 8, no. 10, pp. 5060-5074, 2009.

[13] Z. Wang and G. B. Giannakis, "A simple and general parameterization quantifying performance in fading channels," IEEE Trans. Commun. vol. 51, no. 8, pp. 1389-1398, Aug. 2003. 